\title{
Bend Flow under the Conditions of Curve Intersection Study on Hydraulic Characteristics of the Curve
}

\author{
Meng Jia \\ College of Water Conservancy and Hydropower, Hebei University of Engineering, Water \\ Conservancy Project, Handan, Hebei China
}

410664339@qq.com

Keywords: Curved corners junction; Model test; Inflow angle; Circulation intensity; The ratio of discharge

\begin{abstract}
The flow structure is complex near the curved junction, in previous studies, relatively few studied tributary skew curve river and water sports is more complex due to the centrifugal force under this condition. In order to deeply understand the flow structure based on generalized model test, studied at the different of inflow angle and the ratio of discharge, the flow structure nearby curved corners junction area. Research results show that, when inflow angle is larger, effect of tributaries on the mainstream is getting stronger, vertical average velocity reaches the peak appears at the concave bank in downstream section. The more uneven the distribution of flow velocity becomes with an increase of inflow angle of the overall. When inflow angle becomes larger, Circulation intensity becomes more and more strength, the sections with the largest circulation intensity appears at the concave bank in downstream section is moving downward gradually.
\end{abstract}

\section{曲线交汇条件下弯道水流水力特性试验研究}

\author{
贾猛 \\ 1. 河北工程大学 水电学院、水利工程, 中国 河北 都䣋 056038 \\ a410664339@qq.com
}

摘要: 曲线交汇口附近水流结构复杂, 在以往的研究中, 对支流斜交曲线干流研究相对较少, 而该条件下由于受离心力作用水流运动更为复杂。为探讨该区域水流特性, 通过概化模型试 验, 研究了不同汇流比和入汇角时, 弯道交汇口附近的水流结构特性。得出, 支流入汇角逐 渐增大时, 其对干流影响也随之增强; 交汇口下游断面垂线平均流速出现峰值且断面流速在 交汇口处不均匀性增强; 交汇口附近环流强度随之变大且入汇口下游环流旋度最值断面逐渐 下移。

关键词：曲线型交汇；模型试验；入汇角；环流强度

\section{1. 引言}

交汇型河流在我国各地区水系中普遍存在, 邻近汇流口处水流结构复杂 [1-5], 呈现诸多水动 力学特性: 如支流对干流隹水作用, 交汇口下游出现回流现象, 流速带分布不均等 [6-9]。这 些特性受到很多因素控制, 如交汇口形状、干支流河床高差、河床坡底、入汇角、汇流比等 [10-11], 其中汇流比和入汇角是影响弯道水力特性较为重要的因素。在以往研究中主要是探 讨支流直线交汇于干流的水力学特性 [12], 但支流斜交曲线干流研究相对较少。为此, 本文 通过概化模型对支流交汇于弯道干流凸岸时的弯道水流特性展开研究与分析。 


\section{2. 试验设计}

2.1. 试验模型

水槽整体选用透明有机玻璃, 主支槽进水口分别设有稳流栅, 并设置相应过渡段。模型弯道 圆心角取 $60^{\circ}$, 主槽直线段部分长、宽、高分别为 $4.5 \mathrm{~m} 、 0.6 \mathrm{~m} 、 0.4 \mathrm{~m}$, 曲线部分弧长为 $4 \mathrm{~m}$, 弯曲半径为 $6 \mathrm{~m}$; 支槽长、宽、高分别为 $3 \mathrm{~m} 、 0.3 \mathrm{~m} 、 0.4 \mathrm{~m}$ 。主槽与支槽连接处光滑、角度可调 且不漏水。模型平面布置情况如图 1-1。

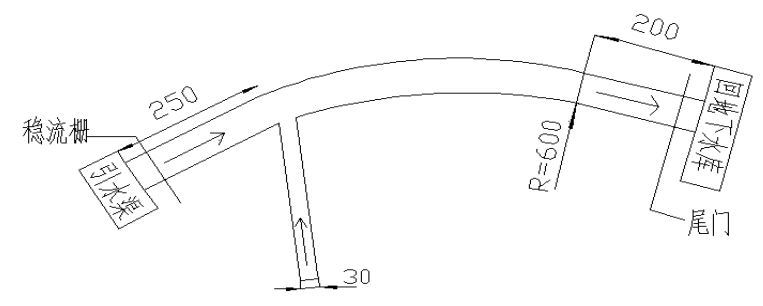

图 1-1 模型平面布置图 (单位: $\mathrm{cm}$ )

2.2. 测点布置及量测设备

本文主要研究, 汇流比一定, 汇流角 $\mathrm{a}$ 为 $45^{\circ} 、 60^{\circ} 、 75^{\circ}$ 时, 曲线型弯道交汇口附近水 流结构变化和垂线流速分布特性。

在弯道段布置流速测点网格, 沿弯道段径向每 $5^{\circ}$ 划分一个断面, 共布置了 13 个测流横断面, 即 $1 、 2 、 3 \cdots \cdots 13$ 。每一个断面从左岸到右岸依次设有 6 个测点, 即测点 1 至测点 6 。具体见 图 1-2。每一条垂线设四个点, 分别为 $0.2 \mathrm{~h} 、 0.4 \mathrm{~h} 、 0.6 \mathrm{~h} 、 0.8 \mathrm{~h}$ ( $\mathrm{h}$ 为每个测点平均水深)。 整个流速网络共有 312 个测点。

流速采用 $\mathrm{ADV}$ 量测, 水深采用测针量测。
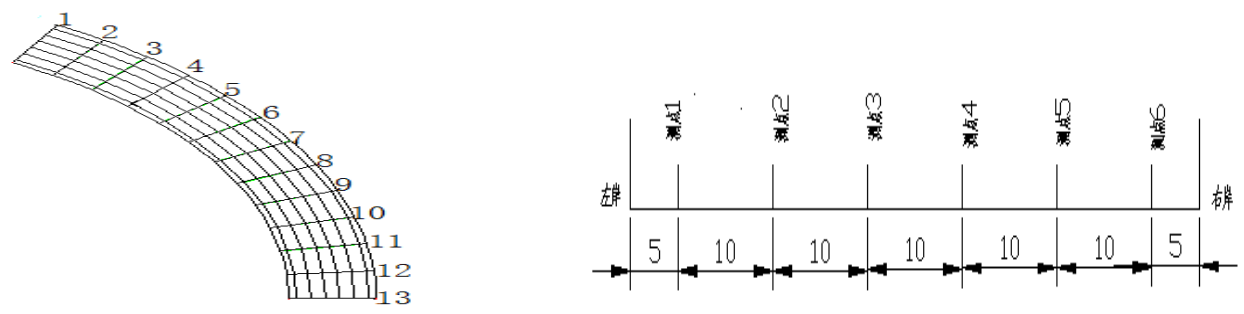

\section{图 1-2 弯道断面测点布置图}

\section{3. 试验结果分析}

\section{1. 弯道环流强度分析}

本试验选取环流旋度值来衡量环流强度的大小, 即横向分速度 $V y$ 和纵向分速度 $V x$ 的比值表 示环流旋度。剖取 $\mathrm{z}=0.2 \mathrm{~h} 、 0.4 \mathrm{~h} 、 0.8 \mathrm{~h}$ 这 3 个流层平面，绘制不同 $\mathrm{a}$ 下断面旋度图。
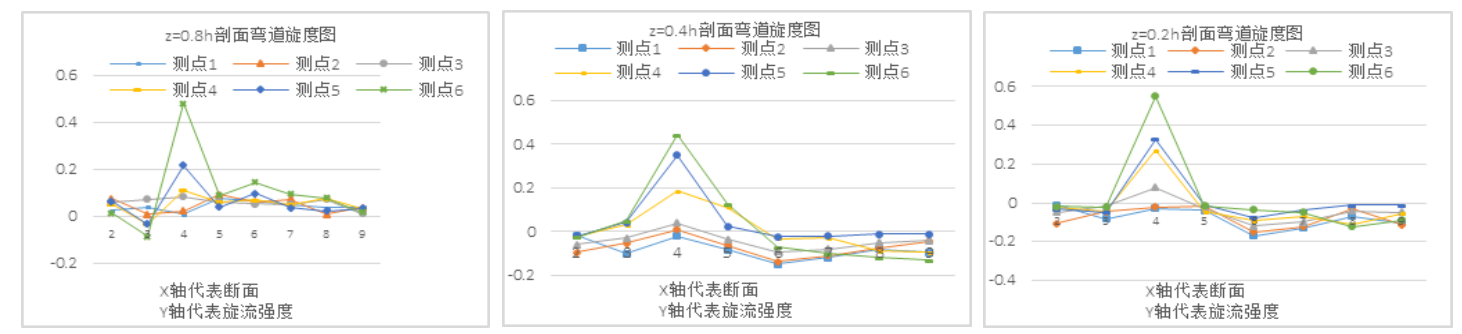

图 2-1 $a$ 为 $45^{\circ}$ 时不同剖面旋流强度图 

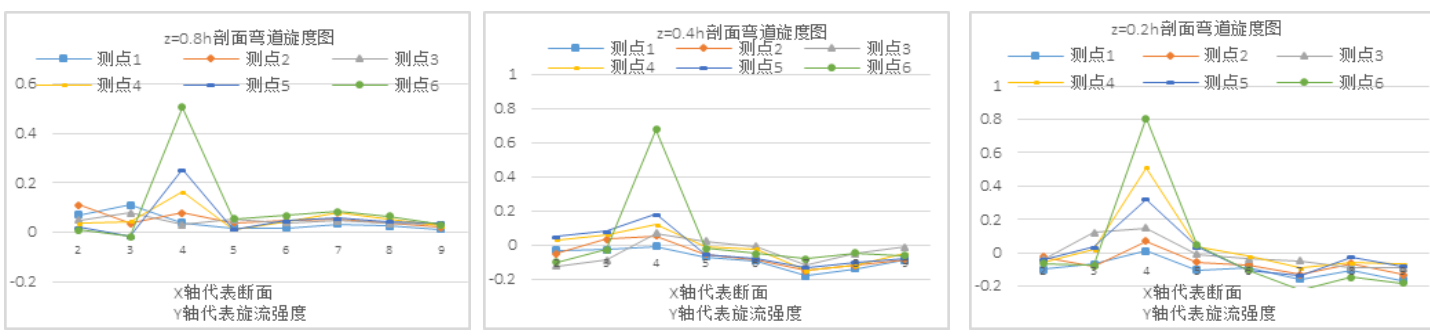

图 2-2 $a$ 为 $60^{\circ}$ 时不同剖面旋流强度图
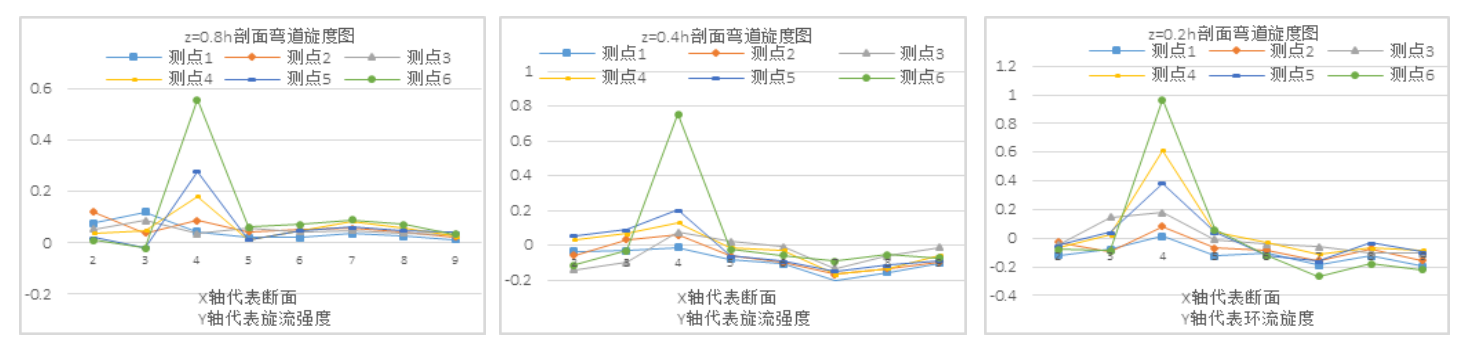

图 2-3 $a$ 为 $75^{\circ}$ 时不同剖面旋流强度图

试验结果表明, 不同剖面旋度值在各断面分布规律不尽相同, 上层旋度略小于底层旋度。在 底层, 水流旋度弯道前半段小于后半段且弯道前半段凹岸侧旋度小于凸岸, 在断面 4 达到峰 值。根据弯道环流理论, 弯道表面水流由凸岸流向凹岸且交汇处受支流顶托作用, 使断面 4 处出现峰值, 由于测点 $1 、 2$ 靠近水槽边壁, 受粘滞性的影响旋度值接近于 0 。弯道进口段水 流较为平顺, 故旋度不大, 环流强度随着水流沿程逐渐增大, 达到顶点后又逐渐减小最终趋 于平稳。

图 2-1 图 2-3 表明, 汇流角是影响环流强度大小的重要因素。当 a 变大时, 支流对主流顶 托作用加强, 使交汇处环流强度加大; 交汇口断面 4 顶托作用最强故出现峰值, 过了交汇口 到交汇口下游, 入汇口下游环流旋度最值断面逐渐下移, 在 $a$ 为 $45^{\circ}$ 时, 入汇口下游出现 环流旋度最值断面是 6 ; 在 $a$ 为 $75^{\circ}$ 时, 入汇口下游出现环流旋度最值断面是 7 。

3.2. 垂线平均流速分析

断面 $2^{\sim} 9$ 为主要交汇区域, 水流交汇特性比较明显, 故选择这 8 个断面为重点研究对象。

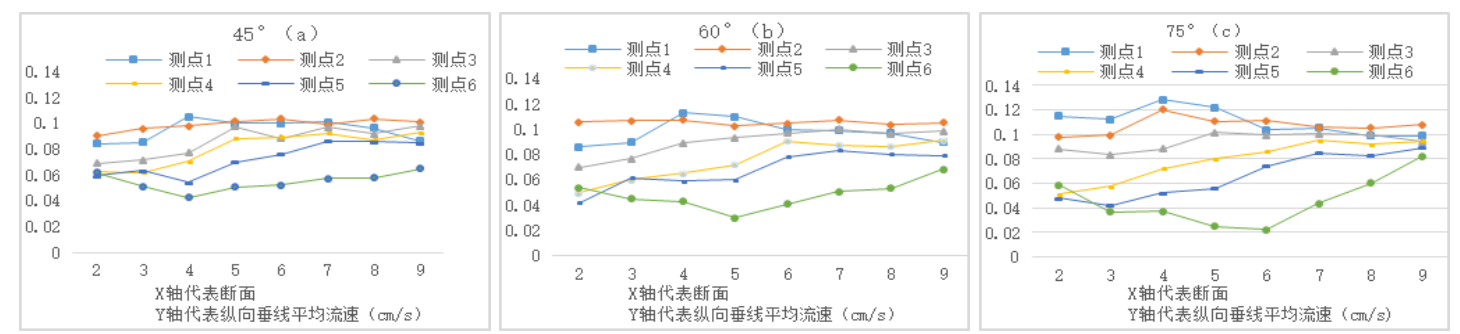

图 2-4 不同 $a$ 时纵向垂线平均流速

通过上述三图对比得出: 入汇支流对主流有挤压作用, 在等汇流比情况下, 随着 a 变大, 支 流对主流影响随之增强, 在交汇口及其下游附近断面出现流速峰值且靠近左岸。紧邻凸岸区 域的流速较低, 这是由于邻近入汇口侧的回流区内水流紊乱, 碰撞产生漩浴, 消耗其能量, 导致其流速减小; 靠近凹岸区域的流速相对凸岸略高, 因为远离入汇侧的区域由于缩小过水 断面使其流速相对较大, 形成收缩区, 当 a 较大时, 支流受惯性作用冲向交汇口对岸, 使主 流受阻缩小过水断面, 加剧了干支流之间碰撞激烈程度, 增强了水流紊动性, 加大了相应能 量损失, 最大流速带在相对应的主流中分布更集中, 所占比例也变大。因此交汇角度变大使 流速分布不均匀。

为分析各特征横断面流速在不同交汇角下变化特性, 引入流速方差。方差越大, 说明流速分 布不均匀性越大。即 


$$
S^{2}=\frac{1}{n}\left[\left(X_{1}-X\right)^{2}+\left(X_{2}-X\right)^{2}+\ldots \ldots+\left(X_{n}-X\right)^{2}\right]
$$

其中, $\mathrm{X}$ 表示纵向垂线平均流速, $\mathrm{n}$ 表示流速样本数量, Xi 表示个体, 而 $\mathrm{S} 2$ 就表示流速方差。

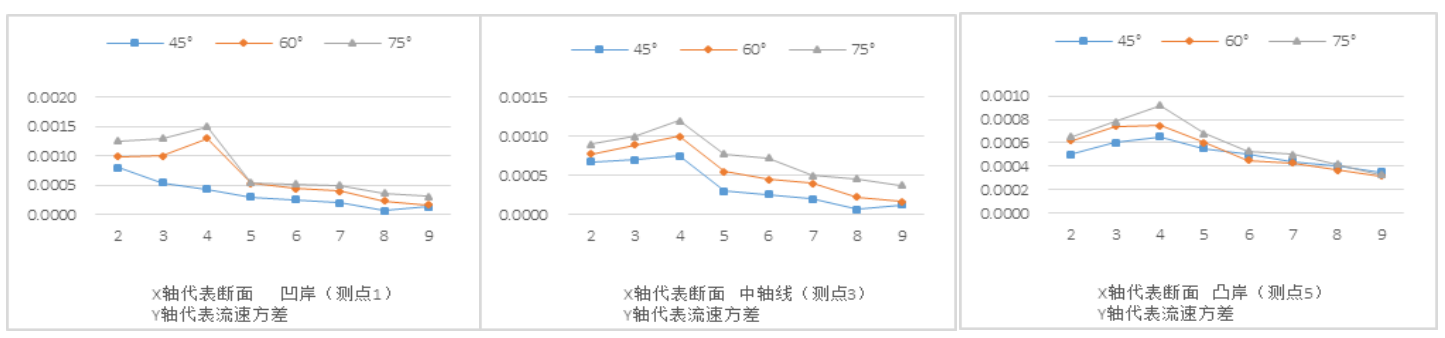

图 2-5 a 不同时各特征横断面流速方差图

由图 2-5 可以看出, a 增大, 交汇口中各特征横断面流速方差也随之增大, 在 $a$ 为 $75^{\circ}$ 时, 流速方差在交汇口处达到峰值。说明 a 增大, 各特征断面流速在交汇口分布不均匀性也增大, 加剧了相应的水流紊动程度。在 $\alpha$ 为 $45^{\circ}$, 凹岸处 (测点 1 ) 的流速方差先减小再趋于稳定。 这是由于较小的交汇角度使主、支流之间交汇相对比较平顺，只是在交汇口处水流碰撞比较 激烈, 但迅速恢复平稳, 对凹岸冲击相对较小。 $\alpha$ 为 $45^{\circ} 、 60^{\circ} 、 75^{\circ}$ 时, 流速方差先增大, 再减小, 最后趋于稳定, 尤其在交汇口（断面 4) 处, 流速方差达到最大值, 说明在交汇口 处流速紊动剧烈, 在下游一段距离后流速再趋于均匀分布。整体上交汇角度越大, 交汇口各 特征横断面流速方差越大，即流速分布的不均匀性越大。

3. 3. 水流动力轴线分析

在之前学者研究的基础上, 认为垂线平均流速是影响水流动力的主要因素, 极大地影响河床 稳定性。因此, 定义河流动力轴线为将沿程各断面垂线平均流速最大值所在位置的连线。
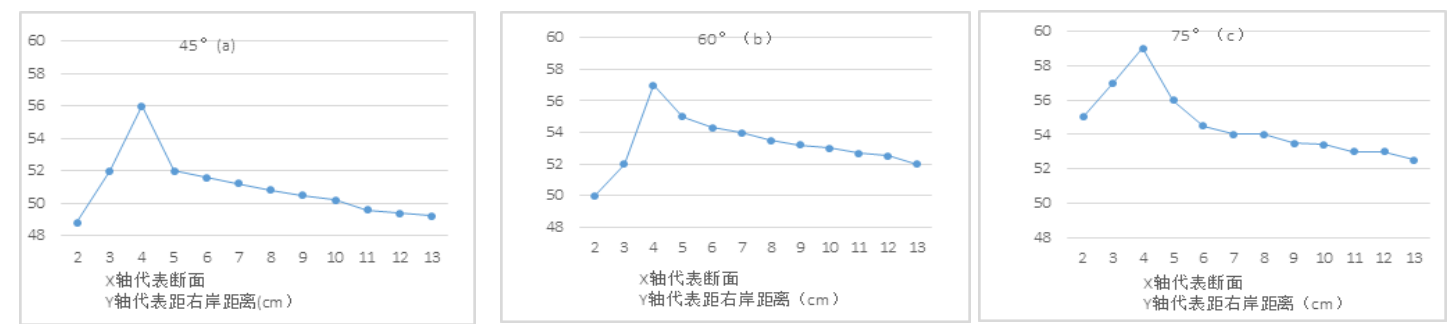

图 2-6 不同 a 下水动力轴线

从图 2-6（c）可以看出，从断面 2 到断面 4 上升较快，在断面 4 处达到峰值，从断面 4 到断 面 5 下降幅度较大, 说明越靠近交汇口, 水动力轴线摆动越剧烈, 变化幅度越大, 由于 (c) 的 a 最大, 所以支流对主流的顶托作用也是最强, 交汇区下游, 从断面 5 至断面 13, 水动 力轴线比较平稳, 交汇区下游一定距离之外的水流流态接近明渠水流, 紧贴左岸。

通过比较可以发现, 当 $\alpha$ 为 $75^{\circ}$ 时, 动力轴线紧贴左岸。对于动力轴线最高点的变化, 发 现随着 $a$ 变大, 动力轴线最高点有向左岸移动趋势。

运用单宽最大动能法对不同 a 下水流动力轴线进行验证。这种方法是将动力轴线看作表征水 流动能的作用线，其垂线单宽功能（Eu）的表达式为:

$E_{u}=0.5 p q v^{2}=K H v^{3}$

上式中： $\rho$ 一水的密度; $q$ 一单宽流量; $H$ 一垂线水深; $v$ 一垂线纵向平均流速; $K$ 一常数。 连接各单宽动能最大值的点即为水流动力轴线。图 2-7 为根据单宽最大动能法绘制的动力轴 线图。 


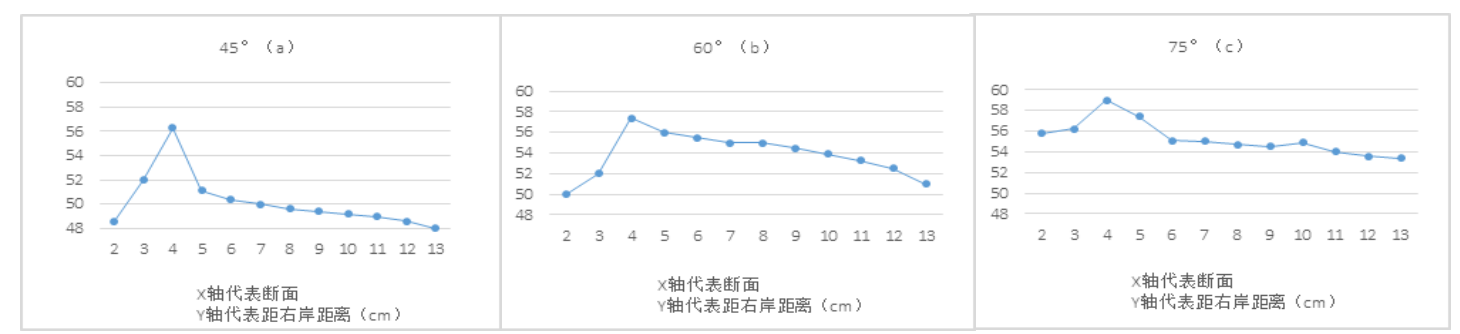

图 2-7 不同 $a$ 下水动力轴线

由图 2-7 得出, 用单宽最大动能法绘制的动力轴线曲线与用垂线最大流速法绘制的动力轴线 曲线走势一致。这并不能说明水深这一因素对动力轴线没有影响, 而是来流量较小, 对交汇 区水流结构影响不大，导致各测量断面的水位波动幅度较小，则根据式 (2-2) 求单宽最大动 能可知, 流速是对交汇区水流动能影响最大的因素, 因此, 采用垂线最大流速法和单宽最大 动能法绘制出的动力轴线曲线基本吻合。

\section{4. 结论}

本文研究了支流曲线入汇干流时不同入汇角下的水流特性。试验表明: 环流强度与汇流角密 切相关。当入汇角逐渐变大, 交汇口附近环流强度也随之变大且入汇口下游环流旋度最值断 面逐渐下移。底层旋度要大于上层旋度。汇流角逐渐变大, 支流对主流顶托影响随之增强, 主支流之间碰撞愈加激烈, 水流紊动越大, 流速分布越不均匀。在交汇口附近垂线平均流速 达到峰值。采用垂线最大流速法和单宽最大动能法绘制出的动力轴线能较好吻合, 动力轴线 最高点有向左岸移动趋势, 故交汇口对岸及其偏下部分应是重点加固部位。

\section{参考文献}

[1] Taylor E. H..Flow characteristics at rectangular open-channel junctions [J]. Trans, ASCE,Vol.109(1944), pp.893-902.

[2] Ramamurthy A. S., Carballada L. B., Tran D. M..Combining open-channel flow at right-angeled junctions [J]. J Hyd Eng, ASCE,Vol.114(1988),No.12,pp.1449-1460.

[3] Hsu C. C., Lee W. J., Chang C. H..Subcritical open channel junction flow [J]. J.Hydr.Eng.ASCE, Vol.124(1998),No.8,pp.847-855.

[4] Lin J. D., Soong H. K..Junction losses in open channel flows [J].Water Resource Research.Vol.15(1979),No.2,pp.414-418.

[5] Gurram S. K..Discussion of "Subcritical open to channel junction flow" [J]. J.Hydr.Eng.ASCE, $2000,126$.

[6] 张挺, 许唯临, 伍平等. $90^{\circ}$ 明渠交汇口三维水力特性数值模拟 [J].水利学报, 2009,40(1): $52-59$

[7] 茅泽育, 赵升伟, 张磊等. 明渠交汇口三维水力特性试验研究[J]. 水利学报, 2004,35(2): 0001-0007.

[8] 周翠英，邓金运. 干支流交汇河段水流泥沙特性研究综述 $[\mathrm{J}]$.水利科技与经济,

[9] 冯亚辉, 郭维东. Y型明渠水流交汇水流数值计算 $[\mathrm{J}]$. 水利水运工程学报, 2006, (4):34-40.

[10]魏文礼, 邵世鹏, 刘玉玲等. 不同交汇角度明渠交汇口三维水力特性的大涡模拟研究 [J]. 应用力学学报, 2015,32(1): 57-63,172. 
[11]刘盛望, 康鹏, 李然等. 水流交汇区的水动力学特性数值模拟 [J]. 水利水电科技进展, 2012,32 (4) : 14-18,22.

[12]刘同宦, 郭炜, 詹否. $90^{\circ}$ 支流入汇区域时均流速分布特征试验研究[J]. 水科学进展, 2009, 20(4): $485-489$.

\section{References}

[1] Taylor E. H..Flow characteristics at rectangular open-channel junctions [J]. Trans, ASCE, Vol.109(1944), pp.893-902.

[2] Ramamurthy A.S., Carballada L. B., Tran D. M..Combining open-channel flow at right-angeled junctions [J]. J Hyd Eng, ASCE,Vol.114(1988),No.12,pp.1449-1460.

[3] Hsu C. C., Lee W. J., Chang C. H..Subcritical open channel junction flow [J]. J. Hydr. Eng. ASCE, Vol.124(1998),No.8,pp.847-855.

[4] Lin J. D., Soong H. K..Junction losses in open channel flows [J].Water Resource Research.Vol.15(1979),No.2,pp.414-418.

[5] Gurram S. K..Discussion of "Subcritical open to channel junction flow" [J]. J. Hydr. Eng. ASCE, 2000,126.

[6] Zhang Ting, Xu Wei-lin, Wu Ping, Mai Dong-ling. "Numerical simulation of three-dimensional characteristics of flow at $90^{\circ}$ open-channel junction" [J]Journal of Hydraulic Engineering, Vol.40(2009),No.1,p.52-59 (in Chinese)

[7] Mao Ze-yu, Zhao Sheng-wei, Zhang Lei, Huang Ji-tang. "Experimental study on 3D flow characteristics at the confluence of open channels" [J]. Journal of Hydraulic Engineering,Vol.35(2004),No.2,p.1-7 (in Chinese)

[8] Zhou Cui-ying,Deng Jin-yun. "Study on the Characteristics of Flow and Sediment at the Confluence of Open Channels"[J].Water Conservancy Science and Technology and Economy, Vol.18(2012),No.8,p.42-45 (in Chinese)

[9] Feng Ya-hui, Guo Wei-dong. "Numerical simulation of flow at Y-shaped open-channel junction" $[\mathrm{J}]$. Hydro-Science and Engineering,(2006),No.4,p.34-40 (in Chinese)

[10] Wei Wen-li, Shao Shi-peng, Liu Yu-ling. "Three-dimensional numerical study of hydraulic characteristics at open-channel junctions with different intersection angles by Large Eddy Simulation", [J]Chinese Journal Of AppliedMechanics,Vol.32(2015),No.1,p.57-63 (in Chinese)

[11]Liu Sheng-yun, Kang Peng, Li Ran, Wei Juan. "A numerical study on hydrodynamic characteristics of confluence flow" [J]. Advances in Science and Technology of Water Resources, Vol.32(2012),No.4,p.14-18 (in Chinese)

[12] Liu Tonghuan, Guo Wei, Zhan Lei. "Experimental study of the velocity profile at $90^{\circ}$ open channel confluence"[J]. Advances in Water Science, Vol.20(2009),No.4,p.485-489 (in Chinese)

作者简介：贾猛 (1990-), 男, 河北唐山, 硕士研究生, 研究方向为水工结构, Emai1:410664339@qq.com 